\title{
Utilização do azul de metileno no tratamento da síndrome vasoplégica após cirurgia cardíaca
}

\author{
José Carlos S. de ANDRADE* ${ }^{\star}$ Mário Lúcio BATISTA FILHO*, Paulo Roberto B. ÉVORA**, \\ José Roberto TAVARES*, Ênio BUFFOLO*, Expedito E. RIBEIRO*, Lélio A. SILVA*, \\ Carlos A. TELES*, Antônia PETRIZZO*, Vitor V. BARATA FILHO*, Renato DUPRAT*
}

RBCCV 44205-297

Andrade J C S, Batista Filho ML, Évora PRB, Tavares J R, Buffolo E, Ribeiro EE, Silva L A, Teles CA, Petrizzo

A, Barata Filho V V, Duprat R - Utilização do azul de metileno no tratamento da síndrome vasoplégica após cirurgia cardiaca. Rev Bras Cir Cardiovasc 1996;11 (2): 107-14.

RESUMO: Relata-se a restauração da resistência vascular sistêmica como uso do azul de metileno (AM) em pacientes submetidos a cirurgia cardiaca com e sem circulação extracorpórea. Todos os pacientes apresentaram no pós-operatório imediato quadro de taquicardia, oligúria, manutenção da perfusão periférica e importante hipotensão arterial sistêmica, refratária a grandes doses de catecolaminas. As avaliaçōes hemodinâmicas pela técnica de termodiluição com cateter de Swan-Ganz monstraram padrão compativel com síndrome vasoplégica, com indice de resistência vascular sistêmica média de 868 dina. $\mathrm{s} . \mathrm{cm}^{5}$, sem resposta a drogas vasoativas. À semelhança do choque endotóxico, a síndrome foi interpretada como decorrente da estimulaçăo da enzima óxido nítricosintetase com conseqüente formação de óxido nítrico (NO) pelas células endoteliais. Utilizou-se então AM, como bloqueador do NO no sistema guanililciclase/guanino-monofosfatociclase, na dose de $1,5 \mathrm{mg} / \mathrm{kg}$ peso, em infusão intravenosa por uma hora. O restabelecimento do tônus vascular sistêmico (IRVS = 1693 dina. $\mathrm{s} . \mathrm{cm}^{5}$ ) com normalização da pressão arterial e do quadro clínico, foi efetivo $e$ rápido, mostrando ser o $\mathrm{AM}$ uma promissora droga na diminuiçāo da morbi-mortalidade da sindrome vasoplégica.

DESCRITORES: Azul de metileno, uso terapêutico. Cirurgia cardiaca, efeitos adversos. Circulação extracorpórea, efeitos adversos. Complicaçōes pós-operatórias, quimioterapia. Hipotensão, quimioterapia. Resistência vascular, efeito de drogas.

\section{INTRODUÇĀO}

A introdução da circulação extracorpórea (CEC) na cirurgia cardíaca permitindo intervenções intracardíacas sob visão direta resultou num rápido progresso na especialidade, dando condiçōes de correção a uma série de cardiopatias até então sem possibilidades de terapêutica cirúrgica.

A utilização rotineira de CEC estimulou o estu- do das complicações a ela associadas, como as alterações verificadas na circulação e hemostasia, bem como no equilíbrio ácido-básico e hidroeletrolítico, mecanismos das trocas gasosas etc, propiciando o aperfeiçoamento do conjunto coração-pulmão artificial.

Apesar da alta qualidade dos equipamentos e materiais hoje empregados na CEC, ainda são freqüentes, nos pacientes a ela submetidos, disfunçōes

Trabalho realizado no Hospital Unicór, São Paulo, SP, Brasil.

Apresentado ao $23^{\circ}$ Congresso Nacional de Cirurgia Cardíaca. Recife, PE, 20 a 23 de março, 1996.

* Do Hospital Unicór

** Do Departamento de Cirurgia, Ortopedia e Traumatologia da Faculdade de Medicina de Ribeirăo Preto.

Endereço para correspondência: José Carlos Andrade. Rua Borges Lagoa, 783, 5ªndar. CEP: 04038-031 São Paulo, SP, Brasil. Tel. [011] 573.3009. 
Andrade J C S, Batista Filho M L, Évora P R B, Tavares J R, Buffolo E, Ribeiro E E, Silva L A, Teles C A, Petrizzo A, Barata Filho V V , Duprat R - Utilização do azul de metileno no tratamento da síndrome vasoplégica após cirurgia cardiaca. Rev Bras Cir Cardiovasc 1996; 11 (2): 107-14.

pulmonares, renais, cerebrais, cardíacas, alteração da coagulação, da homeostase etc, genericamente chamadas de síndrome pós-perfusão (1). Esta síndrome tem sido interpretada como decorrente da ativação do sistema complemento com liberação de enzimas e radicais livres de oxigênio pelos neutrófilos polimorfonucleares, após lesão na barreira endotelial $(1,2)$. Entre as causas determinantes, citam-se: transfusões sangüíneas, utilização de drogas anestésicas, uso do complexo heparina/protamina e até mesmo exposição do sangue a superfícies estranhas não endotelizadas do aparelho coração-pulmão artificial. Além da ativação do complemento dependente do contato sangüíneo com as paredes dos tubos plásticos de cloreto de polivinila (PVC), substâncias a eles incorporadas, como fitalatos, podem também contribuir para as alterações da fisiologia (3).

Uma complicação bem determinada é a síndrome vasoplégica ${ }^{(2)}$, que se caracteriza por hipotensão arterial sistêmica, alto índice cardíaco, baixa pressão capilar pulmonar e insuficiente resposta a catecolaminas, com uma situação hemodinâmica similar à dos pacientes com choque séptico, dada a semeIhança fisiopatológica do processo; isto é, a síndrome vasoplégica seria desencadeada pela produção significativa de indutores da síntese do óxido nítrico, entre eles: fator de necrose tumoral (TNF), interleucina 1 (IL-1) e fator de ativação plaquetária (PAF). Esta produção seria conseqüente à ativação do complemento por anafilatoxinas e endotoxinas, interação de citoquinas induzidas pela adesão molecular, com ativação de polimorfonucleares e liberação de enzimas proteolíticas secundária à degradação de neutrófilos (4-6)

Os pacientes acometidos por esse fenômeno vasoplégico apresentam-se vasodilatados, taquicárdicos, hipotensos, com quadro clínico muito semeIhante ao estado hiperdinâmico da sepse bacteriana. Outra característica observada foi a dificuldade na formação de coágulos com conseqüente sangramento no pós-operatório.

É importante ressaltar que, apesar de periodos prolongados de hipotensão, podem não apresentar comprometimento do sensório, arritmias cardiacas, infarto agudo do miocárdio ou manifestações marcantes de uma coagulação intravascular disseminada, comum na sepse bacteriana.

Estes dados fazem supor a presença de um mediador que produza não só vasoplegia e sangramento, mas também um efeito protetor. Como o óxido nítrico (NO), produzido pelo endotélio, tem características de vasodilatador e antitrombótico endógeno (7), levantou-se a hipótese de que pudesse ser ele o mediador final da sindrome vasoplégica pós-perfusional.
A partir de observação isolada de que o $A M$ elevou a pressão arterial com desaparecimento da vasodilatação periférica, em paciente sem resposta a catecolaminas, concluiu-se pela participação da via arginina-NO e iniciou-se um protocolo para sua utilização na síndrome vasoplégica. Recentes publicações relatam o seu emprego durante a vigência de sepse bacteriana. O AM, promovendo o bloqueio do NO no sistema 'guanilil-ciclase/guanino-monofosfato-ciclase (GC/GMPC), impede o aumento da GMPC, responsável, em última instância, pelo relaxamento da musculatura lisa vascular $(8,9)$.

\section{CASUÍSTICA E MÉTODOS}

Entre 468 pacientes submetidos a cirurgia cardíaca no Hospital' Unicór, durante o ano de 1995, são analisados 6 que apresentaram síndrome vasoplégica no período de pós-operatório imediato.

Constatada a hipotensão arterial, foi instalada monitorização hemodinâmica, à beira do leito, com utilização do cateter de Swan-Ganz.

Comprovada a baixa resistência vascular periférica, foi iniciada administração intravenosa de $A M$ na dosagem de $1,5 \mathrm{mg} / \mathrm{kg}$ de peso corporal, com infusão de metade da dose nos primeiros 15 minutos e o restante até 1 hora.

\section{RELATO DOS CASOS}

\section{Caso de $n^{\circ} 1$}

A.R.M.C., 64 anos, sexo feminino com antecedentes de hipertensão arterial sistêmica e diabetes tipo I, foi submetida a cirurgia de revascularização miocárdica eletiva por quadro de angina estável. Foi realizada anastomose da artéria torácica interna esquerda no ramo interventricular anterior, e enxertos de veia safena para os ramos: interventricular posterior, diagonal e marginal da artéria circunflexa. O período de circulação extracorpórea foi de 117 minutos e o de anoxia de 69 minutos. No pósoperatório imediato desenvolveu hipotensão arterial grave (pressão arterial média de $40 \mathrm{mmHg}$ ) com intensa vasodilatação periférica. Diante da pouca resposta à administração de catecolaminas, optouse pela monitoração hemodinâmica à beira do leito com auxílio de um cateter de Swan-Ganz.

A análise dos parâmetros hemodinâmicos revelou: índice cardiaco (IC) de $3,52 \mathrm{l} / \mathrm{min} / \mathrm{m}^{2}$; indice de resistência vascular periférica (IRVS) de 1090 dina.s. $\mathrm{cm}^{5} / \mathrm{m}^{2}$; índice de trabalho sistólico do ventrículo esquerdo (ITSVE) de $19,8 \mathrm{~g} \cdot \mathrm{min} / \mathrm{m}^{2}$; in- 
Andrade J C S, Batista Filho M L, Évora P R B, Tavares J R, Buffolo E, Ribeiro E E, Silva L A, Teles C A, Petrizzo A, Barata Filho V V, Duprat R - Utilização do azul de metileno no tratamento da síndrome vasoplégica após cirurgia cardiaca. Rev Bras Cir Cardiovasc 1996; 11 (2): 107-14.

dice de trabalho sistólico do ventrículo direito (ITSVD) de $9,5 \mathrm{~g} \cdot \mathrm{min} / \mathrm{m}^{2}$ e pressão capilar pulmonar (PCP) de $11 \mathrm{cmH}_{2} \mathrm{O}$, na vigência de noradrenalina na dose de $0,82 \mathrm{ug} / \mathrm{kg} / \mathrm{min}$.

Após estas determinações, foi administrado ao paciente azul de metileno intravenoso a $5 \%$ diluído em $100 \mathrm{ml}$ de solução fisiológica no período de uma hora, na dosagem de $1,5 \mathrm{mg} / \mathrm{g}$. Foi notória a recuperação progressiva de pressão arterial média com o uso do $\mathrm{AM}$, com conseqüente redução das doses de noradrenalina para $0,08 \mathrm{ug} / \mathrm{kg} / \mathrm{min}(1 / 10$ da dose inicial - Tabela 1).

TABELA 1

VALORES DOS PARAMMETROS OBTIDOS NO CONTROLE PRÉ E PÓS ADMINISTRAÇÃO DE AZUL DE METILENO NO PACIENTE A.R.M.C. COM AS CORRESPONDENTES DOSES DE DROGAS VASOATIVAS

\begin{tabular}{llrr}
\hline PARÂMETRO & UNIDADE & PRÉ & PÓS \\
\hline PAm & mmHg & 60 & 100 \\
FC & b.p.m. & 144 & 80 \\
DC & I.min. & 5,95 & 6,02 \\
IC & I.min $/ \mathrm{m}^{2}$ & 3,52 & 3,56 \\
VS & $\mathrm{ml}$ & 41,3 & 75,2 \\
IVS & $\mathrm{ml} / \mathrm{m}^{2}$ & 24,4 & 44,4 \\
PVC & $\mathrm{cm} \cdot \mathrm{H}_{2} \mathrm{O}$ & 12 & 12 \\
PCP & $\mathrm{cm} \cdot \mathrm{H}_{2} \mathrm{O}$ & 11 & 13 \\
RVS & dina.s $/ \mathrm{cm}^{5}$ & 645 & 1168 \\
IRVS & dina.s $/ \mathrm{cm}^{5} / \mathrm{m}^{2}$ & 1090 & 1973 \\
RVP & dina.s $/ \mathrm{cm}^{5}$ & 241 & 185 \\
IRVP & dina.s $/ \mathrm{cm}^{5} / \mathrm{m}^{2}$ & 407 & 312 \\
TSVE & $\mathrm{g} \cdot \mathrm{min}$ & 33,5 & 102,2 \\
ITSVE & $\mathrm{g} \cdot \mathrm{min} / \mathrm{m}^{2}$ & 19,8 & 30,4 \\
TSVD & $\mathrm{g} \cdot \mathrm{min}$ & 16,1 & 27,6 \\
ITSVD & $\mathrm{g} \cdot \mathrm{min} / \mathrm{m}^{2}$ & 9,5 & 16,3 \\
Dopamina & ug $/ \mathrm{kg} / \mathrm{min}^{2}$ & 20,12 & 2,6 \\
Noradrenalina & ug $/ \mathrm{kg} / \mathrm{min}^{2}$ & 0,82 & 0,08 \\
\hline
\end{tabular}

PAm = pressāo arterial média; $F C=$ freqüência cardiaca; $D C$ = débito cardíaco; $I \mathrm{C}=$ indice cardiaco; VS = volume sistólico; IVS = indice de volume sistólico; PVC = pressão venosa central; PCP = pressāo capilar pulmonar; RVS = resistência vascular sistêmica; IRVS = indice de resistência vascular sistêmica; RVP = resistência vascular pulmonar; IRVP = indice de resistência vascular pulmonar; TSVE = trabalho sistólico do ventrículo esquerdo; ITSVE = indice de trabalho sistólico de ventriculo esquerdo; TSVD = trabalho sistólico de ventrículo direito; ITSVD = indice de trabalho sistólico de ventrículo direito.

\section{Caso de $n^{\circ} 2$}

P.M., 56 anos, sexo masculino, com antecedentes de hipertensão. Atendido com quadro de dissecção aguda da aorta, foi submetido a cirurgia de urgência. Realizou-se reconstrução da aorta ascendente com pericárdio bovino e pexia da valva aórtica. O tempo de perfusão foi de 115 minutos e o de anoxia de 82 minutos. Apresentou hipotensão arterial no pós-operatório imediato (pressão arterial média de $40 \mathrm{mmHg}$ ), com acentuada oligúria. A monitoração hemodinâmica à beira do leito com cateter de Swan-Ganz revelou os seguintes parâmetros: IC de $7,89 \mathrm{l} / \mathrm{min} / \mathrm{m}^{2}$; IRVS de 509 dina.seg. $\mathrm{cm}^{5} / \mathrm{m}^{2}$; ITSVE de 69,5 g. $\mathrm{min} / \mathrm{m}^{2}$; ITSVD de 19,6 g. $\mathrm{min} / \mathrm{m}^{2}$ e pressão capilar pulmonar de $10 \mathrm{cmH}_{2} \mathrm{O}$ em uso de noradrenalina na dose de $0,32 \mathrm{ug} / \mathrm{kg} /$ min. Após a administração de $\mathrm{AM}$, a $5 \%$ diluído em $100 \mathrm{ml}$ de solução fisiológica, em uma hora (dose de $1,5 \mathrm{mg} / \mathrm{kg}$ ) houve boa resposta pressórica, com redução da dose de noradrenalina para $0,06 \mathrm{ug} / \mathrm{kg} /$ min (Tabela 2).

TABELA 2

VALORES DOS PARAMMETROS OBTIDOS NO CONTROLE PRÉ E PÓS ADMINISTRAÇĀO DE AZUL DE METILENO NO PACIENTE P.M, COM AS CORRESPONDENTES DOSES DE DROGAS VASOATIVAS

\begin{tabular}{llrr}
\hline PARÂMETRO & UNIDADE & PRÉ & PÓS \\
\hline PAm & mmHg & 50 & 95 \\
FC & b.p.m & 92 & 100 \\
DC & I.min. & 13,2 & 5,71 \\
IC & I.min.m ${ }^{2}$ & 7,84 & 3,39 \\
VS & $\mathrm{ml}$ & 143,2 & 57,1 \\
IVS & $\mathrm{ml} / \mathrm{m}^{2}$ & 85,2 & 33,9 \\
PVC & $\mathrm{cm} \cdot \mathrm{H}_{2} \mathrm{O}$ & 10 & 7 \\
PCP & $\mathrm{cm} \cdot \mathrm{H}_{2} \mathrm{O}$ & 10 & 8 \\
RVS & dina.s $/ \mathrm{cm}^{5}$ & 303 & 1232 \\
IRVS & dina.s $/ \mathrm{cm}^{5} \cdot \mathrm{m}^{2}$ & 509 & 2069 \\
RVP & dina.s $/ \mathrm{cm}^{5}$ & 42 & 140 \\
IRVP & dina.s $/ \mathrm{cm}^{5} \mathrm{~m}^{2}$ & 70 & 235 \\
TSVE & g.min & 116,8 & 73,7 \\
ITSVE & $\mathrm{g} \cdot \mathrm{min} / \mathrm{m}^{2}$ & 69,5 & 43,8 \\
TSVD & $\mathrm{g} \cdot \mathrm{min}$. & 33 & 13,8 \\
ITSVD & $\mathrm{g} \cdot \mathrm{min} / \mathrm{m}^{2}$ & 19,6 & 8,2 \\
Dopamina & ug $/ \mathrm{kg} / \mathrm{min}^{2}$ & 7,6 & 2,55 \\
Noradrenalina & ug $/ \mathrm{kg} / \mathrm{min}^{2}$ & 0,32 & 0,06 \\
\hline
\end{tabular}

PAm $=$ pressão arterial média; $F C=$ freqũência cardiaca; $D C$ = débito cardíaco; IC = índice cardíaco; VS = volume sistólico; IVS = indice de volume sistólico; PVC = pressão venosa central; $\mathrm{PCP}=$ pressão capilar pulmonar; RVS = resistência vascular sistêmica; IRVS = indice de resistência vascular sistêmica; RVP = resistência vascular pulmonar; IRVP = indice de resistência vascular pulmonar; TSVE = trabalho sistólico do ventriculo esquerdo; ITSVE = indice de trabalho sistólico de ventrículo esquerdo; TSVD = trabalho sistólico de ventrículo direito; ITSVD = índice de trabalho sistólico de ventrículo direito.

\section{Caso de $n \div 3$}

D.D.L., 70 anos, sexo feminino, diabética tipo II, portadora de hipertensão arterial. Foi submetida a revascularização miocárdica eletiva por angina pós infarto, com anastomose da artéria torácica interna esquerda no ramo interventricular anterior e enxertos de veia safena para o ramo marginal da circunflexa e coronária direita, além de plicatura de área acinética do ventrículo esquerdo. O tempo de 
Andrade J C S, Batista Filho M L, Évora P R B, Tavares J R, Buffolo E, Ribeiro E E, Silva L A, Teles C A, Petrizzo A, Barata Filho V V, Duprat R - Utilização do azul de metileno no tratamento da síndrome vasoplégica após cirurgia cardiaca. Rev Bras Cir Cardiovasc 1996; $11(2): 107-14$

perfusão foi de 69 minutos e o de anoxia, de 45 minutos. A paciente evoluiu com hipotensão arterial (pressão arterial média de $50 \mathrm{mmHg}$ ). A monitoração hemodinâmica à beira do leito com cateter de SwanGanz revelou os seguintes parâmetros: IC de 3,75 $\mathrm{I} / \mathrm{min} / \mathrm{m}^{2}$; IRVS de 1071 dina.s. $/ \mathrm{cm}^{5} / \mathrm{m}^{2}$ ITSVE de $37,2 \mathrm{~g} \cdot \mathrm{min} / \mathrm{m}^{2}$; ITSVD de $11,7 \mathrm{~g} / \mathrm{min} / \mathrm{m}^{2}$ e pressão capilar pulmonar de $14 \mathrm{cmH}_{2} \mathrm{O}$, usando dopamina na dose de $7,8 \mathrm{ug} / \mathrm{kg} / \mathrm{min}$. Com a utilização do $A M$ no mesmo esquema terapêutico utilizado nos 2 pacientes anteriores, houve restauração dos níveis pressóricos (Tabela 3 ).

TABELA 3

VALORES DOS PARAMMETROS OBTIDOS NO CONTROLE PRÉ E PÓS ADMINISTRACĀO DE AZUL DE METILENO NO PACIENTE D.D.L, COM AS CORRESPONDENTES DOSES DE DROGAS VASOATIVAS

\begin{tabular}{llrr}
\hline PARÂMETRO & UNIDADE & PRÉ & PÓS \\
\hline PAm & mmHg & 60 & 100 \\
FC & b.p.m. & 80 & 144 \\
DC & l.min & 7,02 & 8,13 \\
IC & I.min $/ \mathrm{m}^{2}$ & 3.65 & 4.23 \\
VS & $\mathrm{ml}$ & 87,7 & 56,4 \\
IVS & $\mathrm{ml} / \mathrm{m}^{2}$ & 45,6 & 29,3 \\
PVC & $\mathrm{cm} \cdot \mathrm{H}_{2} \mathrm{O}$ & 11 & 20 \\
PCP & $\mathrm{cm} \cdot \mathrm{H}_{2} \mathrm{O}$ & 14 & 22 \\
RVS & dina.s $/ \mathrm{cm}^{5}$ & 558 & 688 \\
IRVS & dina.s $/ \mathrm{cm}^{5} / \mathrm{m}^{2}$ & 1071 & 1320 \\
RVP & dina.s $/ \mathrm{cm}^{5}$ & 56 & 98 \\
IRVP & dina.s $/ \mathrm{cm}^{5} / \mathrm{m}^{2}$ & 107 & 188 \\
TSVE & $\mathrm{g} \cdot \mathrm{min}$ & 71,5 & 68,9 \\
ITSVE & $\mathrm{g} \cdot \mathrm{min} / \mathrm{m}^{2}$ & 37,2 & 35,8 \\
TSVD & g.min & 22.5 & 24.4 \\
ITSVD & g.min $/ \mathrm{m}^{2}$ & 11,7 & 12,7 \\
Dopamina & ug $/ \mathrm{kg} / \mathrm{min}^{2}$ & 7,8 & 2,9 \\
Noradrenalina & ug/kg/min & - & - \\
\hline
\end{tabular}

PAm = pressão arterial média; $F C=$ freqũência cardiaca; $D C$ = débito cardiaco; $I \mathrm{IC}=$ indice cardíaco; $\mathrm{VS}=$ volume sistólico; IVS = indice de volume sistólico; PVC = pressão venosa central; $\mathrm{PCP}=$ pressão capilar pulmonar; $\mathrm{RVS}=$ resistência vascular sistêmica; IRVS = índice de resistência vascular sistêmica; RVP = resistência vascular pulmonar; IRVP = indice de resistência vascular pulmonar; TSVE = trabalho sistólico do ventrículo esquerdo; ITSVE = indice de trabalho sistólico de ventrículo esquerdo; TSVD $=$ trabalho sistólico de ventriculo direito; ITSVD = índice de trabalho sistólico de ventriculo direito.

\section{Caso de $n^{\circ} 4$}

C.E.C, 50 anos, sexo masculino, diabético tipo II, com quadro de angina pós infarto do miocárdio recente. Foi submetido a cirurgia eletiva para revascularização do miocárdio com enxertos de veia safena para os ramos interventricular anterior, diagonalis e artéria coronária direita. O tempo de perfusão foi de 74 minutos e o de anoxia, de 65 minutos. Apresentou hipotensão arterial grave no pós-operatório imediato (pressão arterial média de $30 \mathrm{mmHg}$ ).
A monitoração hemodinâmica à beira do leito com cateter de Swan-Ganz revelou os seguintes parâmetros: IC de $5,44 \mathrm{I} / \mathrm{min} / \mathrm{m}^{2}$; IRVS de 396 dina.s./ $\mathrm{cm}^{5} / \mathrm{m}^{2}$; ITSVE de $22,5 \mathrm{~g} \cdot \mathrm{min} / \mathrm{m}^{2}$; ITSVD de 10,8 g. $\mathrm{min} / \mathrm{m}^{2}$ e pressão capilar pulmonar de $10 \mathrm{cmH}_{2} \mathrm{O}$, usando dopamina na dose de $17,9 \mathrm{ug} / \mathrm{kg} / \mathrm{min}$ e noradrenalina na de $0,21 \mathrm{ug} / \mathrm{kg} / \mathrm{min}$. Com utilização do AM no mesmo esquema terapêutico, houve recuperação dos níveis pressórios (Tabela 4 ).

\section{TABELA 4}

VALORES DOS PARÂMETROS OBTIDOS NO CONTROLE PRÉ E PÓS ADMINISTRAÇĀO DE AZUL DE METILENO NO PACIENTE C.E.C, COM AS CORRESPONDENTES DOSES DE DROGAS VASOATIVAS

\begin{tabular}{llrr}
\hline PARÂMETRO & UNIDADE & PRÉ & PÓS \\
\hline PAm & m.jmHg & 35 & 85 \\
FC & b.p.m. & 115 & 115 \\
DC & I.min & 9,8 & 9,02 \\
IC & I.min $/ \mathrm{m}^{2}$ & 5,44 & 5,01 \\
VS & ml & 85,2 & 78,4 \\
IVS & $\mathrm{ml} / \mathrm{m}^{2}$ & 47,3 & 43,5 \\
PVC & $\mathrm{cm} \cdot \mathrm{H}_{2} \mathrm{O}$ & 8 & 11 \\
PCP & $\mathrm{cm} . \mathrm{H}_{2} \mathrm{O}$ & 10 & 32 \\
RVS & dina.s $/ \mathrm{cm}^{5}$ & 229 & 655 \\
IRVS & dina.s $/ \mathrm{cm}^{5} / \mathrm{m}^{2}$ & 396 & 1179 \\
RVP & dina.s $/ \mathrm{cm}^{5}$ & 57 & 70 \\
IRVP & dina.s $/ \mathrm{cm}^{5} / \mathrm{m}^{2}$ & 102 & 126 \\
TSVE & g.min & 40,5 & 90 \\
ITSVE & g.min $/ \mathrm{m}^{2}$ & 22,5 & 50 \\
TSVD & g.min & 19,5 & 42,5 \\
ITSVD & g.min $/ \mathrm{m}^{2}$ & 10,8 & 23,6 \\
Dopamina & ug $/ \mathrm{kg} / \mathrm{min}^{2}$ & 17,9 & 6,4 \\
Noradrenalina & ug $/ \mathrm{kg} / \mathrm{min}^{2}$ & 0,21 & - \\
\hline & & &
\end{tabular}

$\mathrm{PAm}=$ pressāo arterial média; $F C=$ freqũência cardíaca; $D C$ = débito cardiaco; $I \mathrm{C}=$ indice cardiaco; $\mathrm{VS}=$ volume sistólico; IVS = índice de volume sistólico; PVC = pressão venosa central; $\mathrm{PCP}=$ pressão capilar pulmonar; $\mathrm{RVS}$ = resistência vascular sistêmica; IRVS = indice de resistência vascular sistêmica; RVP = resistência vascular pulmonar; IRVP = indice de resistência vascular pulmonar; TSVE = trabalho sistólico de ventrículo esquerdo; ITSVE = indice de trabalho sistólico de ventrículo esquerdo; TSVD = trabalho sistólico de ventriculo direito; ITSVD = indice de trabalho sistólico de ventrículo direito.

\section{Caso de n5}

S.A.C., 57 anos, sexo masculino. Assintomático, com teste ergométrico positivo em controle ambulatorial de hipertensão arterial. Documentada lesão coronariana única em CD, foi tentada angioplastia, sem sucesso (dissecção espiralada da artéria). Realizada operação de urgência de revascularização miocárdica com enxerto de veia safena para $C D$, sem uso de CEC. No pós-operatório imediato, apresentou hipotensão arterial (PAm $=35 \mathrm{mmHg}$ ) não responsiva à administração de volume e de drogas vasoativas (noradrelina e dopamina). Feita monitoração hemodinâmica com cateter de Swan-Ganz e 
Andrade J C S, Batista Filho M L, Évora P R B, Tavares J R, Buffolo E, Ribeiro E E, Silva L A, Teles C A, Petrizzo A, Barata Filho V V, Duprat R - Utilização do azul de metileno no tratamento da síndrome vasoplégica após cirurgia cardíaca. Rev Bras Cir Cardiovasc 1996; 11 (2): 107-14.

administração de AM com restauração dos níveis pressóricos (Tabela 5).

TABELA 5

VALORES DOS PARAMMETROS OBTIDOS NO CONTROLE PRÉ E PÓS ADMINISTRAÇÃO DE AZUL DE METILENO NO PACIENTE S.A.C, COM AS CORRESPONDENTES DOSES DE DROGAS VASOATIVAS

\begin{tabular}{llrr}
\hline PARÂMETRO & UNIDADE & PRÉ & PÓS \\
\hline PAm & mmHg & 50 & 90 \\
FC & b.p.m. & 136 & 112 \\
DC & I.min & 5,88 & 6,92 \\
IC & I.min $/ \mathrm{m}^{2}$ & 3,52 & 4,14 \\
VS & $\mathrm{ml}$ & 43,2 & 55,8 \\
IVS & $\mathrm{ml} / \mathrm{m}^{2}$ & 25,8 & 33,4 \\
PVC & $\mathrm{cm} \cdot \mathrm{H}_{2} \mathrm{O}$ & 4 & 17 \\
PCP & $\mathrm{cm} \cdot \mathrm{H}_{2} \mathrm{O}$ & 5 & 19 \\
RVS & dina.s $/ \mathrm{cm}^{5}$ & 625 & 843 \\
IRVS & dina.s/cm $/ \mathrm{m}^{2}$ & 1043 & 1407 \\
RVP & dina.s $/ \mathrm{cm}^{5}$ & 135 & 138 \\
IRVP & dina.s $/ \mathrm{cm}^{5} / \mathrm{m}^{2}$ & 225 & 230 \\
TSVE & $\mathrm{g} \cdot \mathrm{min}$ & 29,3 & 68,29 \\
ITSVE & g.min $/ \mathrm{m}^{2}$ & 17,5 & 40,8 \\
TSVD & $\mathrm{g} \cdot \mathrm{min}$ & 8,7 & 23,3 \\
ITSVD & $\mathrm{g} \cdot \mathrm{min} / \mathrm{m}^{2}$ & 5,2 & 13.9 \\
Dopamina & ug/kg/min & 2,5 & - \\
Noradrenalina & ug/kg/min & 0,57 & - \\
\hline & & &
\end{tabular}

PAm = pressão arterial média; $F C=$ freqüência cardiaca; $D C$ = débito cardiaco; $\mid \mathrm{C}=$ índice cardíaco; VS = volume sistólico; IVS = índice de volume sistólico; PVC = pressão venosa central; $\mathrm{PCP}=$ pressão capilar pulmonar; RVS = resistência vascular sistêmica; IRVS = índice de resistência vascular sistêmica; RVP = resistência vascular pulmonar; IRVP = indice de resistência vascular pulmonar; TSVE = trabalho sistólico de ventrículo esquerdo; ITSVE = indice de trabalho sistólico de ventrículo esquerdo; TSVD = trabalho sistólico de ventrículo direito; ITSVD = indice de trabalho sistólico de ventrículo direito.

\section{Caso de $n^{\circ} 6$}

M.C.S., 56 anos, sexo feminino, diabética tipo II, assintomática até recente IAM (30 dias) com desenvolvimento de aneurisma ventricular. Realizada revascularização do miocárdio com anastomose da artéria torácica interna para o ramo interventricular anterior e enxertos de vèia safena para o ramo marginal da circunflexa e coronária direita, além de aneurismectomia ventricular. O tempo de perfusão foi de 100 minutos e o de anoxia, de 48 minutos. Evoluiu com hipotensão arterial $\mathrm{Pam}=40 \mathrm{mmHg}$ resistente à administração de dopamina (dose de $14,8 \mathrm{ug} / \mathrm{kg} / \mathrm{min}$ ) e de noradrenalina (dose de 0,28 $\mathrm{ug} / \mathrm{kg} / \mathrm{min}$ ). A monitoração hemodinâmica com cateter de Swan-Ganz constatou os seguintes parâmetros: IC de $3,69 \mathrm{I} / \mathrm{min} / \mathrm{m}^{2}$; IRVS de 1104 dina.s. $\mathrm{cm}^{5} / \mathrm{m}^{2}$; ITSVE de $18,7 \mathrm{~g} . \mathrm{min} / \mathrm{m}^{2}$; ITSVE de $4,9 \mathrm{~g} \cdot \mathrm{min} / \mathrm{m}^{2}$ e pressão capilar pulmonar de $8 \mathrm{~cm}$ de $\mathrm{H}_{2} \mathrm{O}$. A utilização do AM propiciou restabelecimento dos niveis pressóricos e suspensão das drogas vasoativas (Tabela 6 ).
TABELA 6

VALORES DOS PARÂMETROS OBTIDOS NO CONTROLE PRE E PÓS ADMINISTRAÇÃO DE AZUL DE METILENO NO PACIENTE M.C.S, COM AS CORRESPONDENTES DOSES DE DROGAS VASOATIVAS

\begin{tabular}{llrr}
\hline PARÂMETRO & UNIDADE & PRÉ & PÓS \\
\hline PAm & mmHg & 60 & 100 \\
FC & b.p.m. & 160 & 128 \\
DC & I.min & 5,54 & 3,00 \\
IC & I.min $/ \mathrm{m}^{2}$ & 3,69 & 2,00 \\
VS & $\mathrm{ml}$ & 34,6 & 23,4 \\
IVS & $\mathrm{ml} / \mathrm{m}^{2}$ & 23,0 & 15,6 \\
PVC & $\mathrm{cm} \cdot \mathrm{H}_{2} \mathrm{O}$ & 9 & 18 \\
PCP & $\mathrm{cm} \cdot \mathrm{H}_{2} \mathrm{O}$ & 8 & 16 \\
RVS & dina.s $/ \mathrm{cm}^{5}$ & 736 & 1919 \\
IRVS & dina.s $/ \mathrm{cm}^{5} / \mathrm{m}^{2}$ & 1104 & 2878 \\
RVP & dina.s $/ \mathrm{cm}^{5}$ & 115 & 186 \\
IRVP & dina.s $/ \mathrm{cm}^{5} / \mathrm{m}^{2}$ & 172 & 279 \\
TSVE & $\mathrm{g} \cdot \mathrm{min}$ & 28,1 & 28,5 \\
ITSVE & $\mathrm{g} \cdot \mathrm{min} / \mathrm{m}^{2}$ & 18,7 & 19,0 \\
TSVD & $\mathrm{g} \cdot \mathrm{min}$ & 7,4 & 3,6 \\
ITSVD & $\mathrm{g} \cdot \mathrm{min} / \mathrm{m}^{2}$ & 4,9 & 2.4 \\
Dopamina & ug $/ \mathrm{kg} / \mathrm{min}^{2}$ & 14,8 & 2,9 \\
Noradrenalina & ug $/ \mathrm{kg} / \mathrm{min}^{2}$ & 0,28 & - \\
\hline
\end{tabular}

PAm = pressão arterial média; $F C$ = freqũência cardíaca; $D C$ = débito cardiaco; $I \mathrm{C}=$ índice cardiaco; VS = volume sistólico; IVS = indice de volume sistólico; PVC = pressão venosa central; $\mathrm{PCP}=$ pressão capilar pulmonar; $\mathrm{RVS}=$ resistência vascular sistêmica; IRVS = índice de resistência vascular sistêmica; RVP = resistência vascular pulmonar; IRVP = indice de resistência vascular pulmonar; TSVE = trabalho sistólico de ventrículo esquerdo; ITSVE = indice de trabalho sistólico de ventrículo esquerdo; TSVD = trabalho sistólico de ventrículo direito; ITSVD = índice de trabalho sistólico de ventrículo direito.

\section{COMENTÁRIOS}

A presença do endotélio como condição para um vaso sangüíneo relaxar sob a ação da acetilcolina foi estabelecida por FURCHGOTT \& ZAVADZKI (10), em 1980. A interpretação deste fenômeno biológico levou à proposição de que o endotélio produziria um fator que foi denominado "fator relaxante derivado do endotélio" (EDRF - endothelium-derived relaxing factor). Durante 5 anos, considerou-se apenas que este fator não era um prostanóide e era difusível e, a partir de 1985, com o ínicio dos métodos de cultura de células endoteliais, foi possível a manipulação bioquímica e farmacológica do mesmo, constatando-se que sua ação era muito semelhante à dos vasodilatadores coronarianos. Com estas observações, FURCHGOTT \& ZAWADZKI (10) e IGNARRO et al. (11) propuseram que o EDRF seria o óxido nítrico (NO). Posteriormente, PALMER et al (12), do grupo de Moncada, concluindo que o endotélio vascular produzia o óxido nítrico tendo como fonte endógena orgânica a L-arginina, iniciaram uma nova era no estudo das moléstias cardiovasculares e da reatividade vascular. 
Andrade J C S, Batista Filho M L, Évora P R B, Tavares J R, Buffolo E, Ribeiro E E, Silva L A, Teles C A, Petrizzo A, Barata Filho V V, Duprat R - Utilização do azul de metileno no tratamento da síndrome vasoplégica após cirurgia cardiaca. Rev Bras Cir Cardiovasc 1996; 11 (2): 107-14.

Durante o período de evolução desses conhecimentos, criaram-se ferramentas farmacológicas para o estudo da liberação e efeitos do NO, especialmente dos seus bloqueadores: a hemoglobina (como scavenger do NO); o azul de metileno como bloqueador da guanilato-ciclase; L-NMMA (n-monometil-l-arginina), L-NOARG (n-nitroarginina), L-NAME (n-nitro-l-argininametil ester) como bloqueadores da síntese do NO $(7,13)$.

A fisiopatologia do choque endotóxico com vasodilatação arterial sistêmica é hoje exaustivamente estudada. Casos com agressão endotelial, lesão por isquemia, ação de endotoxinas liberadas por bactérias Gram negativas (lipopolissacárides), são entendidos como uma síndrome de reação inflamatória sistêmica de proporções e conseqüências variáveis, desde uma simples granulocitose e febre a um clássico choque séptico. As endotoxinas ou a ativação do complemento por lesão endotelial teriam a propriedade de liberar citoquinas, substâncias tais como: TNF, IL-1 e PAF. Entre outras, estas citoquinas seriam responsáveis diretas pela indução da enzima óxido nítrico sintetase (NOS) que levaria à produção de óxido nítrico pelas células endoteliais e conseqüente vasodilatação, hiporreatividade vascular, disfunção miocárdica, inibição da respiração celular e, em casos mais graves, até insuficiência de múltiplos órgãos e sistemas (13).

Usado por muitos anos no tratamento da metahemoglobinemia e da intoxicação causada por nitratos, o azul de metileno (AM), em trabalhos experimentais e clínicos também mostrou uma resposta adequada na reversão da hipotensão arterial sistêmica no choque endotóxico provocado pela ação de endotoxinas $(3,8,9)$. O AM teria esta ação por diminuir os níveis de guanilil-ciclase com conseqüente quebra do sistema GC/GMPC por onde é mediada a ação do óxido nítrico.

Trabalhos experimentais mostram a ação do AM restaurando a reatividade aórtica a níveis controle em ratos com choque endotóxico induzido ${ }^{(14)}$. Em 1992, o AM foi citado como restaurador da resistência vascular sistêmica em 2 pacientes com choque séptico (9). Resposta semelhante foi obtida recentemente (1995) em outra série de 14 pacientes na mesma condição clínica (8). Assim, seria possivel obter efeitos benéficos não só sobre a vasodilatação, mas também na diminuição do sangramento cirúrgico pela ação do NO sobre a função plaquetária, sem afetar os efeitos imunológicos e a microcirculação. Esses casos relatados na literatura com o uso de $\mathrm{AM}$ em choque séptico mostraram uma ação efetiva, porém não duradoura, no aumento da resistência vascular sistêmica.

Em nossos casos, a ação, além de efetiva, foi duradoura com retorno da reatividade arterial à catecolamina e manutenção da pressão arterial. Esta evolução diferente poderia ser explicada por uma persistente produção de indutores de óxido nítricosintetase na presença da sepse, o que não ocorreria nos pacientes apenas submetidos à CEC e/ou ao uso do complexo heparina-protamina, nos quais não há uma persistência, mas só uma transitoriedade de produção de citoquinas.

A síndrome vasoplégica, que, no Serviço, apresentou incidência de $1,2 \%$ ( 6 casos em 468 pacientes), tem alta morbidade e mortalidade quando acomete pacientes submetidos a cirurgia cardiaca $(1,2)$. Além da necessidade do seu pronto reconhecimento, exige precisão terapêutica. Erros de interpretação podem levar a situações de hiper-hidratação com a administração generosa de liqüidos na tentativa de reversão, da hipotensão sistêmica e capilar pulmonar. Além de não agir na causa básica geradora da sindrome - a baixa resistência vascular sistêmica - essas atitudes podem agravar uma situação já de alto risco, caracterizada por edema intersticial e pulmonar secundários a alterações primárias da permeabilidade capilar. Com isto, o tratamento da síndrome vasoplégica tem sido, até hoje, baseado na utilização de altas doses de noradrenalina.

Nos casos apresentados, com a utilização do AM, foi possivel diminuir substancialmente as doses de catecolaminas reduzindo, portanto, o risco de eventuais complicações decorrentes do seu uso.

A opção pelo uso do AM deveu-se ao fato de ser medicação já utilizada na clínica, enquanto que os bloqueadores da síntese do NO, além de não serem adotados para utilização terapêutica, são associados a lesões celulares importantes. À exceção da dexametasona, todos os outros bloqueadores de síntese conhecidos atuam não só na ação da óxido nítrico-sintetase induzível (NO-si) como também na expressão da óxido nítrico-sintetase constitutiva (NO-sc). A NO-sc, responsável pela produção basal e estimulada do NO pelo endotélio, e tono vascular fisiológico, deve ser preservada. Por outro lado a NO-si, responsável pela produção de NO nos tecidos, associada a lesões celulares e disfunçōes orgânicas, deve ser inibida. Portanto, a utilização do AM é atraente pelo fato de não interferir na síntese fisiológica do NO pela NO-sc. Sua ação ocorreria ao nível do alvo do NO, ou seja, na musculatura lisa vascular, impedindo a ativação da guanilil-ciclase e, com isso, diminuindo os níveis de GMPC.

Embora a amostra seja pequena, a aplicação de estatística (ANOVA) (Gráfico 1) mostrou que as diferenças antes e após a utilização do AM foram significantes para os valores do aumento da PAm e do IRVS, mas não significantes para as diferenças 
Andrade J C S, Batista Filho M L, Évora P R B, Tavares J R, Buffolo E, Ribeiro E E, Silva L A, Teles C A, Petrizzo A, Barata Filho V V, Duprat R - Utilização do azul de metileno no tratamento da síndrome vasoplégica após cirurgia cardíaca. Rev Bras Cir Cardiovasc 1996; $11(2): 107-14$.

\section{GRÁFICO 1}

REPRESENTAČÃO GRÁFICA DAS MÉDIAS E DESVIOS. PADRŌES DOS VALORES OBTIDOS ANTES E APÓSA UTILIZACCAOO DO AZUL DE METILENO NO TRATAMENTO DA SINDROME VASOPLÉGICA.
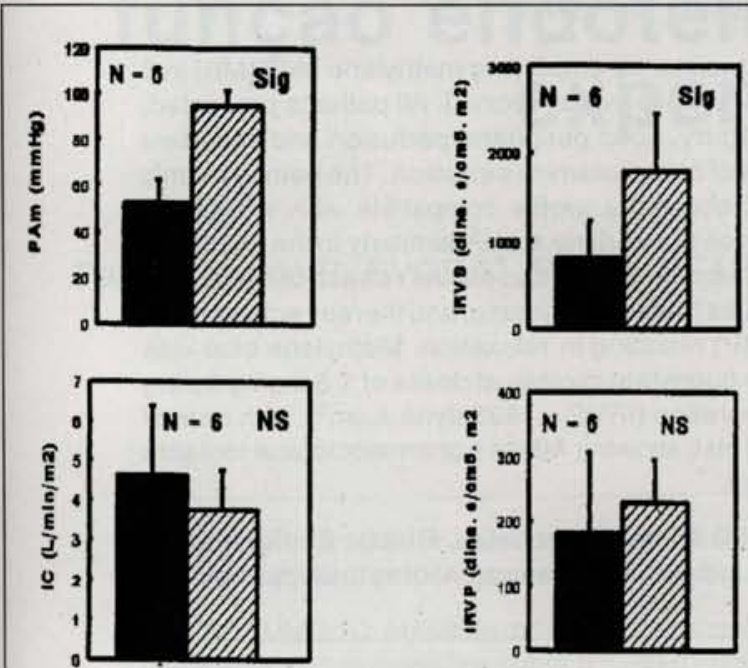

\section{AZUL DE METILENO $(1,5 \mathrm{mg} / \mathrm{kg})$}

PAm $=$ pressāo arterial média; IRVS = indice da resistência vascular sistêmica; IC = indice cardiaco; IRVP = indice da resistência vascular pulmonar; ANOVA $(P<0.05)$; Sig = significante; $N S=$ não significante.

entre os valores do IC e do IRVP. Isto sugere que a ação do $A M$ se faz principalmente ao nível do território arteriolar onde a produção de NO é maior.
O fato da administração do $A M$ não afetar de modo significante o DC, em contraposição à inibição da síntese de NO pelo L-NMMA, é mais um fato a favor da sua utilização. Estes dados são superponiveis a recente relato que, em 9 pacientes com choque séptico, mostrou com administração de $\mathrm{AM}$, uma melhora da pressão arterial acompanhada de aumento do débito cardíaco (15).

É evidente que este campo de pesquisa exige novas investigações para melhor entendimento dos complexos mecanismos envolvidos na gênese da síndrome vasoplégica. Para que se possa atuar profilaticamente, talvez o desenvolvimento dos fatores anticitoquinas visando à prevenção do desencadeamento da cascata da reação inflamatória seja uma linha atraente obtendo-se, assim, maior segurança e melhor resultado com a utilização da CEC. Por outro lado, a ocorrência da vasoplegia em 1 paciente, que foi submetido a revascularização do miocárdio sem o auxílio de CEC (Caso de no 5), levanta a possibilidade de que os fenômenos desencadeadores da liberação de NO não estejam relacionados somente a condições relacionadas com o desvio cardiopulmonar.

Assim, o azul de metileno, atuando sobre o tônus vascular através do bloqueio da ação do NO na musculatura lisa vascular (GMPC), se não reverte totalmente o distúrbio vasotônico, ao menos diminui de maneira significativa a necessidade do uso de catecolaminas como a noradrenalina e, por conseqüência, seus efeitos colaterais, contribuindo substancialmente para a diminuição da morbi-mortalidade na síndrome vasoplégica. 
Andrade J C S, Batista Filho M L, Évora P R B, Tavares J R, Buffolo E, Ribeiro E E, Silva L A, Teles C A, Petrizzo A, Barata Filho V V, Duprat R - Utilização do azul de metileno no tratamento da síndrome vasoplégica após cirurgia cardíaca. Rev Bras Cir Cardiovasc 1996; 11 (2): 107-14.

Andrade J CS, Batista Filho ML, Évora PRB, Tavares J R, Buffolo E, Ribeiro EE, Silva LA, Teles C A, Petrizzo A, Barata Filho V V, Duprat R - Methylene blue administration in the treatment of the vasoplegic syndrome after cardiac surgery. Rev Bras Cir Cardiovasc 1996; 11 (2): 107-14.

ABSTRACT: The restoration of the systemic vascular resistance employing methylene blue (MB) in 6 patients after cardiac surgery with and without cardiopulmonary bypass is reported. All patients presented, during the immediate postoperative period, tachycardia, oligury, good peripheral perfusion and important systemic arterial hypotension not responsible to large doses of catecholamines infusion. The hemodynamic analysis, using the Swan-Ganz thermodilution technique, showed a profile compatible with vasoplegic syndrome, with the mean index of systemic vascular resistance of 868 dyne.s.cm ${ }^{5}$, similarly to the endotoxic shock this syndrome was assumed to be caused by nitric oxide synthase stimulation and release of nitric oxide (NO), by endothelial cells. Once released, NO stimulates solube guanylate cyclase, and thereby activates the production of cyclic guanosine $3^{\prime}, 5^{\prime}$ monophosphate (cGMP) resulting in relaxation. Methylene blue was administered to block the $\mathrm{NO}$ action by inhibiting the soluble guanylate cyclase at doses of $1.5 \mathrm{mg} / \mathrm{kg}$ during one hour intravenous infusion. Systemic vascular tone restoration (IRVS $=1693$ dyne.s.cm ${ }^{5}$ ), with normal arterial pressure and clinical performance, were effective and fast, showing MB as a pharmacological tool able to reduce morbidity and mortality of the vasoplegic syndrome.

DESCRIPTORS: Methylene blue, therapeutic use. Heart surgery, adverse effects. Extracorporeal circulation, adverse effects. Postoperative complications, drug therapy. Hypotension, drug therapy. Vascular resistance, drug effects.

\section{REFERÊNCIAS BIBLIOGRÁFICAS}

1 Gomes W J - Ativação leucocitária e circulação extracorpórea. Arq Bras Cardiol 1993; 60: 3-4.

2 Gonçalves Jr. I, Carvalho A C, Castello $\mathrm{H} \mathrm{J}$ et al. Vasodilatação periférica no pós-operatório de cirurgia cardíaca: sindrome vasoplégica. Arq Bras Cardiol 1992; 59 (Supl. 2): 84.

3 Mayer B, Brunner F, Schmidt K - Inhibition of nitric oxide synthesis by methylene blue. Biochem Pharmac 1993; 45: 367-74.

4 Andersen L W, Baek L, Degn $\mathrm{H}$, et al. - Presence of circulating endotoxins during cardiac operations. Thorac Cardiovasc. Surg 1987; 93: 1159.

5 Menasché $\mathrm{P}$, Peynet $\mathrm{J}$, Larivière $\mathrm{J}$ et al. - Does normothermic during cardiopulmonary bypass increase neutrophil-endothelium interactions? Circulation 1994; 90 (Part 2): 275-9.

6 Rocke D A, Gaffin S L, Wells M T, Koen Y, Brock-Utine $\mathrm{J} \mathrm{G}$ - Endotoxemia associated with cardiopulmonary bypass. Thorac Cardiovasc Surg 1987; 93: 832-7.

7 Évora P R B - O impacto científico da descoberta do óxido nítrico como vasodilatador e antitrombótico endógeno (Editorial). Arq Bras Cardiol 1993; 61:3-5.

8 Preiser J C, Lejeune P, Roman A et al. - Methylene blue administration in septic shock: a clinical trial. Crit Care Med 1995; 23: 259-64.
9 Shneider F, Lutun P H, Hasselmann M, Stoclet J C, Tempé J D - Methylene blue increases systemic vascular resistance in human septic shock: preliminary observations. Int Care Med 1992; 18: 309-11.

10 Furchgott R F \& Zawadzki J V - The obligatory role of endothelial cells in the relaxation of arterial smooth muscle to acetylcholine. Nature 1980; 228: 373-6.

11 Ignarro L J, Buga G M, Wood K S, Byrness R E, Chaudhuri $\mathrm{G}$ - Endothelium-derived relaxing factor produced and released from artery and vein is nitric oxide. Proc Natl Acad Sci USA 1987; 84: 9265-9.

12 Palmer R M J, Ashton D S, Moncada S - Vascular endothelial cells synthesize nitric oxide from Larginine. Nature 1988; 333: 664-6.

13 FINK M P (Guest ed.) - Nitric oxide. New horizonts: the science and practice of acute medicine 1995; 3: 1-138.

14 Paya D, Gray G A, Stocllet J C - Effects of methylene blue on blood pressure and reactivity to norepinephrine in endotoxemia rats. J Cardiovasc Pharmac 1993; 21:926-30.

15 Daemen-Gubbels C R G H, Groeneveld P H P, Groeneveld A B J, von Kamp G J, Bronsveld W, Thijs L G - Methylene blue increases myocardial function in septic shock. Crit Care Med 1995; 23: 1363-70. 